ELECTRONIC RESEARCH ANNOUNCEMENTS OF THE AMERICAN MATHEMATICAL SOCIETY

Volume 12, Pages 95-99 (August 2, 2006)

S $1079-6762(06) 00166-1$

\title{
INTRINSIC HARNACK ESTIMATES FOR NONNEGATIVE LOCAL SOLUTIONS OF DEGENERATE PARABOLIC EQUATIONS
}

\author{
EMMANUELE DIBENEDETTO, UGO GIANAZZA, AND VINCENZO VESPRI
}

(Communicated by Luis A. Caffarelli)

\begin{abstract}
We establish the intrinsic Harnack inequality for nonnegative solutions of the parabolic $p$-Laplacian equation by a proof that uses neither the comparison principle nor explicit self-similar solutions. The significance is that the proof applies to quasilinear $p$-Laplacian-type equations, thereby solving a long-standing problem in the theory of degenerate parabolic equations.
\end{abstract}

\section{MAin RESUlts}

Let $E$ be an open set in $\mathbb{R}^{N}$, and for $T>0$, let $E_{T}$ denote the cylindrical domain $E \times(0, T]$. Consider quasi-linear, parabolic differential equations of the form

$$
u_{t}-\operatorname{div} \mathbf{A}(x, t, u, D u)=b(x, t, u, D u) \quad \text { weakly in } E_{T},
$$

where the functions $\mathbf{A}: E_{T} \times \mathbb{R}^{N+1} \rightarrow \mathbb{R}^{N}$ and $b: E_{T} \times \mathbb{R}^{N+1} \rightarrow \mathbb{R}$ are only assumed to be measurable and subject to the structure conditions

$$
\left\{\begin{array}{l}
\mathbf{A}(x, t, u, D u) \cdot D u \geq C_{o}|D u|^{p}-C^{p} \\
|\mathbf{A}(x, t, u, D u)| \leq C_{1}|D u|^{p-1}+C^{p-1}, \\
|b(x, t, u, D u)| \leq C|D u|^{p-1}+C^{p-1}
\end{array} \quad \text { a.e. in } E_{T},\right.
$$

where $p \geq 2$ and $C_{o}$ and $C_{1}$ are given positive constants, and $C$ is a given nonnegative constant. A function

$$
u \in C_{\mathrm{loc}}\left(0, T ; L_{\mathrm{loc}}^{2}(E)\right) \cap L_{\mathrm{loc}}^{p}\left(0, T ; W_{\mathrm{loc}}^{1, p}(E)\right)
$$

is a local weak solution to (1.1) if for every compact set $K \subset E$ and every subinterval $\left[t_{1}, t_{2}\right] \subset(0, T]$,

$$
\begin{aligned}
\left.\int_{K} u \varphi d x\right|_{t_{1}} ^{t_{2}}+\int_{t_{1}}^{t_{2}} \int_{K}[ & \left.-u \varphi_{t}+\mathbf{A}(x, t, u, D u) \cdot D \varphi\right] d x d t \\
& =\int_{t_{1}}^{t_{2}} \int_{K} b(x, t, u, D u) \varphi d x d t
\end{aligned}
$$

for all bounded test functions

$$
\varphi \in W_{\mathrm{loc}}^{1,2}\left(0, T ; L^{2}(K)\right) \cap L_{\mathrm{loc}}^{p}\left(0, T ; W_{o}^{1, p}(K)\right) .
$$

Received by the editors January 20, 2006.

2000 Mathematics Subject Classification. Primary 35K65, 35B65; Secondary 35B45.

Key words and phrases. Degenerate parabolic equations, Harnack estimates, Hölder continuity.

(C)2006 American Mathematical Society Reverts to public domain 28 years from publication 
The parameters $\left\{N, p, C_{o}, C_{1}, C\right\}$ are the data, and we say that a generic constant $\gamma=\gamma\left(N, p, C_{o}, C_{1}, C\right)$ depends upon the data if it can be quantitatively determined a priori only in terms of the indicated quantities.

For $\rho>0$, let $B_{\rho}$ be the ball of radius $\rho$ centered at the origin in $\mathbb{R}^{N}$, and for $y \in \mathbb{R}^{N}$, let $B_{\rho}(y)$ denote the homothetic ball centered at $y$. For $\theta>0$, set also

$$
Q_{\rho}(\theta)=B_{\rho} \times(-\theta, \theta]
$$

and for $(y, s) \in \mathbb{R}^{N} \times \mathbb{R}$,

$$
(y, s)+Q_{\rho}(\theta)=B_{\rho}(y) \times(s-\theta, s+\theta] .
$$

Local weak solutions to (1.1) - (1.5) are locally bounded and locally Hölder continuous in $E_{T}([3)$. This fact was used to prove the Harnack inequality, but unfortunately only in some special instances. We can now show that the Harnack estimate actually holds in full generality and independently of the Hölder continuity.

Theorem 1.1 (Intrinsic Harnack Inequality). Let $u$ be a nonnegative weak solution to (1.1) - 1.5 . There exist positive constants $c$ and $\gamma$ depending only upon the data, such that for almost all $\left(x_{o}, t_{o}\right) \in E_{T}$ and all cylinders $\left(x_{o}, t_{o}\right)+Q_{2 \rho}(4 \theta) \subset E_{T}$,

$$
u\left(x_{o}, t_{o}\right) \leq \gamma\left[\inf _{B_{\rho}\left(x_{o}\right)} u\left(x, t_{o}+\theta\right)+C \rho\right], \quad \theta=\left(\frac{c}{u\left(x_{o}, t_{o}\right)}\right)^{p-2} \rho^{p}
$$

where $C$ is the same as in (1.2). As a consequence, any locally bounded weak solution to (1.1)-(1.2) is locally Hölder continuous in $E_{T}$, and thus (1.6) permits an independent proof of the Hölder continuity of solutions established in [3].

In (1.6) the time $\theta$ is intrinsic to the solution $u$ and to the geometry of the ball $B_{\rho}\left(x_{o}\right)$. It would be desirable to have an estimate where the space-time geometry can be prescribed a priori, independently of $u\left(x_{o}, t_{o}\right)$. This will be the object of a future research.

\section{Novelty AND SIGNIFICANCE}

Equation (1.1) with the structure conditions (1.2) is a quasi-linear version of the degenerate, homogeneous equation

$$
u_{t}-\sum_{i, j=1}^{N} D_{x_{j}}\left(|D u|^{p-2} a_{i j}(x, t) D_{x_{i}} u\right)=0 \quad \text { weakly in } E_{T},
$$

where the coefficients $a_{i j}$ are measurable and locally bounded in $E_{T}$ and the matrix $\left(a_{i j}\right)$ is almost everywhere positive definite in $E_{T}$. If $\left(a_{i j}\right)=\mathbb{I}$, then (2.1) reduces to the degenerate, prototype parabolic $p$-Laplace equation

$$
u_{t}-\operatorname{div}\left(|D u|^{p-2} D u\right)=0 \quad \text { weakly in } E_{T} .
$$

Both (2.1) and (2.2) satisfy the structure conditions (1.2) with $C=0$. Accordingly, nonnegative weak solutions of these equations satisfy the intrinsic Harnack inequality (1.6) with $C=0$. 
2.1. The linear case $p=2$. The Harnack inequality for local, nonnegative solutions of the heat equation ([1.6), with $p=2$ and $C=0$ ) was established independently by Hadamard [6] and Pini [8], using local representation of solutions in terms of heat potentials. In [9, Moser established the same Harnack inequality for weak solutions of (2.1) for $p=2$, using energy-based, measure-theoretical arguments. Moser's proof is nonlinear in nature, and it can be extended almost verbatim ([10, 1]) to the quasi-linear versions (1.1)-(1.2) with $p=2$. At almost the same time, Ladyzhenskaya, Solonnikov, and Ural'tseva [7] established, by means of DeGiorgi-type measure-theoretical arguments, that weak solutions of such quasilinear equations (still for $p=2$ ) are locally bounded and locally Hölder continuous. It turns out that the Harnack inequality of Moser can be used to establish the Hölder continuity of solutions. On the other hand, it was observed in 2] that the Hölder continuity implies the Harnack inequality for nonnegative solutions.

Thus a summary of the quasi-linear theory for the "linear" case $p=2$ is that the Hölder continuity and Harnack inequality for nonnegative solutions are mutually equivalent. However, establishing either of them independently requires independent measure-theoretical arguments.

2.2. The degenerate case $p>2$. Neither Moser's nor DeGiorgi's ideas, in the version of [7], seem to apply when $p \neq 2$, even for the prototype case (2.2). Some progress has been made using the idea of time-intrinsic geometry, in which the time is scaled, roughly speaking, by $u^{p-2}$. This permits establishing that weak solutions of (1.1)-(1.2), for all $p>1$, are Hölder continuous in $E_{T}$ ([3], Chapters III and IV). It was also observed that, while the Harnack inequality in the Moser form is in general false for $p>2$, it might hold in this time-intrinsic geometry. Indeed, it was shown that (1.6) with $C=0$ holds for nonnegative solutions of (2.2). The proof is based on the maximum principle and comparison functions constructed as variants of the Barenblatt similarity solutions (see [3], Chapter VI, for an account of the theory),

$$
\Gamma_{p}(x, t)=\frac{1}{t^{N / \lambda}}\left[1-\gamma_{p}\left(\frac{|x|}{t^{1 / \lambda}}\right)^{\frac{p}{p-1}}\right]_{+}^{\frac{p-1}{p-2}}, \quad t>0,
$$

where

$$
\gamma_{p}=\left(\frac{1}{\lambda}\right)^{\frac{1}{p-1}} \frac{p-2}{p}, \quad \lambda=N(p-2)+p .
$$

As $p \rightarrow 2$, this tends pointwise to the fundamental solution of the heat equation. In this sense, $\Gamma_{p}$ is some sort of $p$-heat potential. Thus this approach can be regarded as paralleling that of Hadamard and Pini for the heat equation.

The novelty of Theorem 1.1 lies in producing a proof of the Harnack inequality (1.6) based only on measure-theoretical arguments. This bypasses any notion of maximum principle and potentials, and permits an extension to nonnegative solutions of quasi-linear equations of the type of (1.1)-(1.2). Its significance is in paralleling Moser's measure-theoretical, quasi-linear development, following Hadamard and Pini's potential representations for the heat equation. Moreover, our approach does not use any kind of covering argument and the cross-over lemma, which were used in Moser's proof but can be regarded as rather artificial. 
In [5] we will give a detailed proof of Theorem 1.1, built on measure-theoretical facts established in [4. Particular care will be taken in showing how the Harnack inequality implies the Hölder continuity of the solution.

Thus a summary of the quasi-linear theory, for the "degenerate" case $p>2$, is that the Hölder continuity and intrinsic Harnack inequality are mutually equivalent. However, establishing either of them independently requires independent measuretheoretical arguments. Finally, as $p \rightarrow 2$, the intrinsic Harnack inequality (1.6) and the corresponding Hölder theory recover Moser's classical estimate and the corresponding Hölder estimates of [7].

2.3. Expansion of positivity. The main technical novelty is illustrated by referring back to the "linear" case $p=2$.

Let $u$ be a nonnegative, local solution of the heat equation in $E_{T}$. Suppose that $B_{\rho}(y) \times\left(s-\rho^{2}, s+4 \rho^{2}\right] \subset E_{T}$ and

$$
\operatorname{meas}\left\{x \in B_{\rho}(y) \mid u\left(x, s-\rho^{2}\right)<M\right\}<\alpha \operatorname{meas}\left\{B_{\rho}\right\}
$$

for some $M>0$ and some $\alpha \in(0,1)$. Then there exists $\eta=\eta(\alpha) \in(0,1)$ such that for all $x \in B_{2 \rho}(y)$,

$$
u\left(x, s+4 \rho^{2}\right) \geq \eta M .
$$

Thus information on the measure of the "positivity set" of $u$ at the time level $s-\rho^{2}$, over the ball $B_{\rho}(y)$, translates into an expansion of the positivity set both in space (from $B_{\rho}(y)$ to $B_{2 \rho}(y)$ ), and in time (from $s-\rho^{2}$ to $s+4 \rho^{2}$ ). This fact continues to hold for quasi-linear versions of the heat equation and was established in [2].

A similar fact for $p>2$ is in general false, as one can verify from the Barenblatt solution (2.3) - (2.4). The main technical novelty of our investigation is that a similar fact continues to hold for the degenerate equations (1.1)-(1.2), in a time-intrinsic geometry. Precisely,

Lemma 2.1. Let $u$ be a nonnegative, local, weak solution of (1.1)-(1.2). There exist positive constants $\gamma$ and $b$, and $\eta \in(0,1)$, depending only upon the data and independent of $(y, s), \rho$, and $M$, such that if

$$
u(x, s) \geq M \quad \text { for all } x \in B_{\rho}(y)
$$

then either $M<\gamma C \rho$, or for a.e. $x \in B_{2 \rho}(y)$,

$$
u(x, t) \geq \eta M \quad \text { with } t=s+\left(\frac{b}{\eta M}\right)^{p-2}(4 \rho)^{p} .
$$

\section{REFERENCES}

1. D. G. Aronson and J. Serrin, Local behaviour of solutions of quasi-linear parabolic equations, Arch. Rat. Mech. Anal. 25 (1967), 81-123. MR0244638 (39:5952)

2. E. DiBenedetto, Harnack estimates in certain function classes, Atti Sem. Mat. Fis. Univ. Modena 37 (1989), 173-182. MR0994063 (90k:35044)

3. E. DiBenedetto, Degenerate Parabolic Equations, Springer-Verlag, Series Universitext, New York, 1993. MR1230384 (94h:35130)

4. E. DiBenedetto, U. Gianazza, and V. Vespri, Local clustering on the nonzero set of functions in $W_{l o c}^{1,1}(E)$, Rend. Lincei Mat. Appl. 17 (2006), 223-225.

5. E. DiBenedetto, U. Gianazza, and V. Vespri, Harnack estimates for quasilinear degenerate parabolic differential equations, in preparation.

6. J. Hadamard, Extension à l'équation de la chaleur d'un théorème de A. Harnack, Rend. Circ. Mat. di Palermo, Ser. 2, 3 (1954), 337-346. MR0068713 (16:930a) 
7. O. A. Ladyzenskaya, N. A. Solonnikov, and N. N. Ural'tseva, Linear and Quasilinear Equations of Parabolic Type, Transl. Math. Monographs, 23, Amer. Math. Soc., Providence, RI, 1968. MR0241822 (39:3159b)

8. B. Pini, Sulla soluzione generalizzata di Wiener per il primo problema di valori al contorno nel caso parabolico, Rend. Sem. Mat. Univ. Padova 23 (1954), 422-434. MR0065794 (16:485c)

9. J. Moser, A Harnack inequality for parabolic differential equations, Comm. Pure Appl. Math. 17 (1964), 101-134. MR0159139 (28:2357)

10. N. S. Trudinger, Pointwise estimates and quasi-linear parabolic equations, Comm. Pure Appl. Math. 21 (1968), 205-226. MR0226168(37:1758)

Department of Mathematics, Vanderbilt University, 1326 Stevenson Center, NashVILLE, TN 37240, USA

E-mail address: em.diben@vanderbilt.edu

Dipartimento di Matematica "F. Casorati", Università di Pavia, via Ferrata 1, 27100 Pavia, Italy

E-mail address: gianazza@imati.cnr.it

Dipartimento di Matematica "U. Dini", Università di Firenze, viale Morgagni 67/A, 50134 FiRENZE, ITALY

E-mail address: vespri@math.unifi.it 